\title{
Effectiveness of Flax Seed Pillow on Stress and Physical Parameters of Premature Infants
}

\author{
Tessy Thomas ${ }^{1}$, Sujatha $\mathbf{R}^{2}$ \\ ${ }^{1}$ Professor \& HOD, ${ }^{2}$ MSc Nursing Student, Department of Child Health Nursing, Nitte Usha Institute of Nursing \\ Sciences, Nitte University, Paneer, Derlakatte, Mangalore, Karnataka
}

\begin{abstract}
Introduction : As per WHO report in 2018, every year, 15 million babies are born prematurely, the leading cause of child deaths under age five every year. Many survivors of preterm birth face a lifetime of disability, including learning disabilities and visual and hearing problems.
\end{abstract}

Purpose of the study: To find the effectiveness of a flaxseed pillow on stress and physical parameters in premature infants.

Methodology : An evaluative two group pretest post-test approach selected for the study. Premature infants selected by a purposive sampling technique and applied with a flaxseed pillow. All the infants were observed two times per day for five consecutive days from the day of recruitment. Stress and Physical parameters obtained and analyzed to find the effectiveness of the intervention.

Results: Out of 34 Premature infants, 17 (50\%) underwent flax seed pillow treatment, and remaining were the control group. Independent sample t-test results showed that there is a significant difference (pre-post) in the stress score between the experimental and control group. The paired t-test result revealed that there is a difference $(\mathrm{p}<0.05)$ in the stress score among the experimental groups for all five days.

Conclusion: An increasing number of premature birth is one of the most significant issues faced by the country today. Many developmental care interventions provided to preterm babies, which are cost-effective care and help these vulnerable in their phase of prematurity. Flaxseed pillow was effective in reducing the stress of premature infants in the NICU.

Keywords: Intensive Care Units, Neonatal, Low Birth Weight, Infant, Premature, Flax, Seeds

\section{Introduction}

A premature baby is one born before 37 completed weeks of gestation. Prematurity refers to the inability of the infant who is born before the usual gestational period to adapt to extrauterine existence in comparison to the survival rate in mature infants. Prematurity usually carries a high rate of morbidity and mortality unless optimal care is given to maintain life. Prematurely born infants face multiple challenges and obstacles on their path to continued growth and development, and

\section{Corresponding Author:}

Sujatha R

Email ID:- sujathakannappan@gmail.com

ORCID Number- 0000-0003-3942-0849 they struggle to adapt to the unnatural environment of the Neonatal Intensive Care Unit (NICU). Some challenges are directly related to differences between the environment of the uterus and in the NICU. The unexpected stressors from various stimuli, individually or in combination, can cause pain and lead to fatigue. In this hostile environment, the premature infant is forced to use more of limited resources to cope with the environment rather than to grow and develop.

Prematurity is a stage where infants find it difficult to adapt to the external noxious environment of the tertiary care unit, where they are exposed to continuous stressors of the external world. Premature infants experience an environment that is rife with a negative touch. These stressors got a detrimental effect on the growth of the 
premature infant since these infants have difficulty in adapting to life outside the uterus as a result of immature body systems.

Developmental care is an approach that uses a range of medical and nursing interventions that aim to decrease the stress of premature infants in NICU. The developmentally appropriate care for the premature neonate enables the growth and development to its maximum. ${ }^{1}$

Researchers studied the effects of neuro developmental sensory stimulations and they documented weight gain and early discharge, as a result of these sensory enhancement interventions. ${ }^{2}$

As a result of the literature investigations, the researcher recognized that an opportunity existed to explore the effectiveness of a new, inexpensive, low technology developmental intervention that is a flaxseed pillow. Application of flax seed pillow reduces the stressors and improves the stability of premature infants, which helps in the effective utilization of resources for growth and development, resulting in weight gain. Flaxseed pillow uses natural, tiny, flat, smooth seeds that give the pillow the flexibility to form to any shape. Because of the seed's oil content, the pillows keep the heat or cold for up to 2 hours. When heated and applied to the body Flax Seed Pillows release a comforting, moist heat. These are very beneficial in soothing sore and tight muscles, Produce a calming effect and help curb insomnia, Act as warming pads - they heat cold hands and feet. It also helps poor circulation \& calms the mind. Flaxseed pillow refers to a pillow that is filled with flax seeds used under the scapula of babies in order to rejuvenate the body by providing continuous warmth and a massaging effect when the seeds slide over one another during the body movements.

The study aims to assess the effect of a flaxseed pillow on stress and physical parameters in premature infants.

\section{Method}

An evaluative approach with the Pre-test Post-test control group design, which is quasi-experimental, was adopted. The research proposal was placed in the Institutional ethics committee and obtained permission. (NUINS/CON/NU/IEC/ 2014-15 dated 26/09/2014). In this study, Premature infants who are medically stable, born between the gestational age of 32 to 36 weeks, who does not require any other support, except warmer, IV fluids or/and full enteral feedings were included. Premature infants who are falling above 90th percentile $\&$ below the 10th percentile in the growth chart require surgery or respiratory support, and any genetic anomalies and congenital malformations infants excluded from this study.

\section{Data collection tools}

The data was collected by demographic proforma for mother and premature infants, Premature infant stress behavior assessed by observation checklist, and weight

a) Demographic Proforma for premature infants comprised of Gestational age, Gender, Order of birth, Birth weight, the weight of the baby for five consecutive days, Time of discharge, and duration of hospital stay.

Demographic Proforma for mother includes age, type of delivery, indication for preterm delivery, medical conditions present during the antenatal period, administration of any medications during the antenatal period.

b) Premature infant stress behavior observation checklist used to assess the level of stress. The observation checklist for the study developed based on a theory called the Synactive theory of infant development, which is proposed by Dr. Als. The disorganized behavior of the premature infants in various subsystems (autonomic/ physiological, motor behaviors, state behaviors, selfregulatory behaviors) of the Synactive theory of newborn behavioral organization and development. It includes motor changes like, (flexion of arms and legs, arms salute etc), autonomic changes (color changes, breathing and heart rate variations etc), states behavior (gaze averting, staring, etc), and self-regulatory behavior (change in position, grasping, sucking). ${ }^{3}$

The observation checklist consists of 18 parameters under these subsystems, which describes a premature infant "s behavior in NICU, classified into organized and disorganized behavior. The score indicates the stress of premature infants. The maximum score is 18 , and the minimum score is 1 . Nine subject experts of nursing tested the content validity and relevance of the checklist. The reliability items in the tool were summed up and the total stress score of the day obtained. Then the intraclass correlation the coefficient was calculated, which was 0.715 , and the $p$-value is 0.009 , which weighs the reliability of the tool 0.85 . 
c) Calibrated infant weighing machine used to measure weight (kilogram).

\section{Data Collection Process}

In the NICU, the premature infants born between the age group of 32-36 weeks of gestation were selected by purposive sampling. The purpose of the study explained to the parents, and confidentiality was assured and informed written consent taken. Demographic Proforma was filled initially after that each premature infant was observed for 1 hour, and pretesting is done by filling the observation checklist by the researcher. The premature infants divided into two groups, experimental and control group consisting of 17 subjects each. After the pretesting, the experimental group received 30 minutes of flax seed pillow treatment, whereas the control group did not receive it. The flaxseed pillow is kept over the upper back of the babies( over the scapula). After the treatment for the experimental group, both the groups were subjected to a post-test for 1-hour observation with an observation checklist. The experimental group received five consecutive days of treatment with a flaxseed pillow and control group without a pillow. Each subject was weighed all the five days and the weight on the discharge day, and the duration of hospital stay also recorded

\section{Data Analysis}

The collected information summarized by using descriptive statistics. ( Frequency, Percentage, Mean \& Standard deviation) .

The effectiveness of flaxseed pillow on stress and physical parameters assessed by using Paired " $t$ " test.

Chi-square \& Fisher "s exact test used to find the association between the selected demographic variables with physical parameters and stress.
The difference in duration of hospital stay, physical parameters, and stress between the subject with flax seed pillow treatment and without treatment compared by using independent " $t$ " tests. The level of significance for this study is $5 \%$.

\section{Results}

\section{Description of sample characteristics}

About $18(52.9 \%)$ of premature babies are males, and female babies are $16(47.1 \%)$. Most of the premature babies $24(70.6 \%)$ belong to the gestational age group of 32-34 weeks, and only 10 (29.4) among 34 premature infant belongs to 34-36 weeks of gestation. Among 34 premature infants, $15(44.1 \%)$ were second-born, $10(29.4 \%)$ were firstborn, $7(20.6 \%)$, and $2(5.9 \%)$ were third and fourth born, respectively.

About 15 (44.1\%) of the mothers of preterm infants are in between the age group of 25-29 followed by 13 $(38.2 \%)$ are in $20-24,3(8.8 \%)$ are in $<20,2(5.9 \%)$ of them in 30 to 34 and only 1 person (2.9\%) in 35-40 years. Around half of the deliveries were normal 18 (52.9\%), others were LSCS 10(29.4\%), Vacuum 4(11.8\%), and only $2(5.9 \%)$ were forceps delivery. Premature rupture of the membrane was the most common cause for preterm delivery $8(23.5 \%)$; the second cause is fetal distress, which constituted 6(17.6\%). Placental abnormalities $4(11.8 \%)$ and pre-eclampsia $4(11.8 \%)$ were the third common cause, which is followed by multiple pregnancies $3(8.8 \%)$. Two of the common diseases present during the antenatal period were Hypertension $8(23.5 \%)$ and Gestational Diabetes mellitus 7(20.6\%). Mothers with other diseases like Oligohydramnios, Polyhydramnios, and Cardiac conditions constituted about 3(8.8\%), 4(11.8\%), and 3(8.8\%), respectively. Among 34 mothers, 8 (23.5\%) of them were on regular medication for medical conditions.

Table:- 1 The effectiveness of the flaxseed pillow on stress and physical parameters within the groups by using the Paired "to" test. $\quad n=34$

\begin{tabular}{|l|l|l|l|l|}
\hline Within Group comparison & Day & Mean difference & t value & P value \\
\hline \multirow{5}{*}{ Experimental group } & Day 1 & 2.03 & 4.81 & $<0.001^{*}$ \\
\cline { 2 - 6 } & Day 2 & 2.85 & 7.45 & $<0.001^{*}$ \\
\cline { 2 - 6 } & Day 3 & 2.00 & 4.57 & $<0.001^{*}$ \\
\cline { 2 - 5 } & Day 4 & 1.76 & 3.95 & $<0.001^{*}$ \\
\cline { 2 - 5 } & Day 5 & 1.47 & 4.92 & $<0.001^{*}$ \\
\hline
\end{tabular}


Cont ... Table:- 1 The effectiveness of the flaxseed pillow on stress and physical parameters within the groups by using the Paired " $t$ " test.

\begin{tabular}{|l|l|l|l|l|}
\hline & Day 1 & 0.35 & 0.98 & 0.339 \\
\cline { 2 - 6 } Control group & Day 2 & 0.02 & 0.08 & 0.935 \\
\cline { 2 - 6 } & Day 3 & 0.82 & 2.69 & $0.016^{*}$ \\
\cline { 2 - 6 } & Day 4 & 1.00 & 3.51 & $0.003^{*}$ \\
\cline { 2 - 5 } & Day 5 & 0.38 & 1.04 & 0.311 \\
\hline
\end{tabular}

(* significant)

It is evident that the calculated $t$ values are more than the table value on all the five consecutive days of the experimental group and the $\mathrm{p}$ values are $<0.001$ on all the five days. It indicates that a flaxseed pillow is effective in reducing the level of stress among premature infants at a $5 \%$ level of significance.

Table 2:- The difference in the level of stress between the experimental and control group in each day respective of the intervention. (Independent sample t-test) $\quad \mathrm{n}=34$

\begin{tabular}{|l|l|l|l|l|}
\hline \multicolumn{2}{|l}{ Day } & $\begin{array}{l}\text { Mean difference between the } \\
\text { experimental and control group }\end{array}$ & t value & P-value \\
\hline \multirow{2}{*}{ Day 1 } & Pre & 1.08 & 1.69 & 0.105 \\
\cline { 2 - 6 } & Post & 2.76 & 4.81 & $<0.001^{*}$ \\
\hline \multirow{5}{*}{ Day 2 } & Pre & 0.61 & 1.00 & 0.322 \\
\cline { 2 - 6 } & Post & 3.44 & 6.02 & $<0.001^{*}$ \\
\hline \multirow{2}{*}{ Day 3 } & Pre & 1.55 & 3.58 & $<0.001^{*}$ \\
\cline { 2 - 6 } & Post & 2.73 & 5.17 & $<0.001^{*}$ \\
\hline \multirow{2}{*}{ Day 4 } & Pre & 1.52 & 3.63 & $<0.001^{*}$ \\
\cline { 2 - 6 } & Post & 2.29 & 4.21 & $<0.001^{*}$ \\
\hline \multirow{2}{*}{ Day 5 } & Pre & 2.11 & 4.16 & $<0.001^{*}$ \\
\hline & Post & 2.11 & & $<0.001^{*}$ \\
\hline
\end{tabular}

The data presented in table 2, shows that except the baseline measurements of day $1(t=1.69)$ and day $2(t=1)$ calculated values are more than the table value 2.04 , also the $p$ values are $<0.05$. Hence there is a difference in stress level between experiment and control group at a five percent level of significance except day 1 and day 2. Hence 
the null hypothesis (H01) for stress is rejected, and the research hypothesis (H1) for stress is accepted.

\section{Discussion}

The findings of the present study show that premature rupture of the membrane was the common cause for preterm delivery $8(23.5 \%)$; the second cause is fetal distress, which constituted $17.6 \%$. Placental abnormalities $4(11.8 \%)$ and pre-eclampsia $4(11.8 \%)$ were the third common cause, which is followed by multiple pregnancies $3(8.8 \%)$. The finding is supported by the study conducted on Epidemiology of preterm premature rupture of fetal membranes (PPROM) at the University Teaching Hospital, Lusaka which shows premature rupture of the membrane is the common cause of preterm births. ${ }^{4}$

Similar findings can be seen in various studies which showed that PROM is associated with $30-40 \%$ of preterm deliveries and is the identifiable leading cause of preterm delivery.

The present study showed that $29 \quad(85.29 \%)$ mothers of premature infants among 34 , had medical illness during the antenatal period like Gestational Diabetes Mellitus 7 (20.6\%), Hypertension 8 (23.5\%), Oligohydramnios and cardiac conditions 3 each (8.8\%), Polyhydramnios $4(11.8 \%)$ and other conditions 4 (11.8\%) respectively. The present study also shows that two of the common diseases present during the antenatal period were hypertension $8(23.5 \%)$ and Gestational Diabetes mellitus 7(20.6\%).

The present study is supported by the study findings on hypertension which showed that hypertension is the most common medical disorder of pregnancy and is reported to complicate up to 1 in 10 gestations and affects an estimated 2,40,000 women in the United States every year.

Findings of a study done in Western Australia on antenatal risk factors also support that hypertension (13.3\%) and gestational diabetes $(8.2 \%)$ are the two common diseases that exert risks on the antenatal period. This finding reveals that whenever a mother is in the high-risk pregnancy, she is most likely to deliver a premature infant. ${ }^{5}$

In the present study, results showed that in the experimental group the mean score of stress before keeping flax seed pillow was higher compared to the score after the treatment with a flaxseed pillow for the five consecutive days. This study is consistent with the findings where the flaxseed pillow is used as a new developmental approach.

This study is supported based on the efficacy of another study on the soothability of preterm infants, which is done in the US, where the preterm infants are soothed after the treatment with a flaxseed pillow. ${ }^{6}$

This study does not show any statistical differences in weight gain and duration of hospital stay between experimental and control group. This may be due to the small sample size and duration of flax seed application. Even though there is no marked difference, still the treatment could establish one day difference in duration of hospital stay and $0.4 \mathrm{~g}$ of weight difference between the experimental and control group.

Similar result found in a study where, Infants in the treatment group also gained more weight in the first 3 weeks of life and spent more time awake. Discharge weights and length of stay at hospital did not vary between the two groups. ${ }^{7}$

From the above findings, the researchers felt that developmental care techniques like massage and tactile stimulation had got a beneficial effect on stress behaviors. It is also evident that flaxseed pillow, which is a similar developmental care measure like massage and tactile stimulation, has got a stress-reducing effect on the hospitalized premature infants because flaxseed pillow provides a similar tactile stimulation as that of massage on the body of premature infants when the infant is placed over the pillow.

The limitations of this study were data collected from only one NICU, the researchers recruited only AGA babies and the study assessed only a few parameters of the subsystems of the Synactive theory of newborn behavior and development.

The implications of the study are nurses working in NICU should be able to recognize the stress cues or stress responses of the preterm babies to decrease the duration of hospital stay, and the nurses are responsible for providing education to parents of preterm infants regarding their management at home, inculcating the developmental care measures.

\section{Conclusion}

An increasing number of premature birth is one 
of the most significant issues faced by the country today. To help these vulnerable people in their phase of prematurity nursing profession has come with many developmental care interventions were preterm babies are provided the best possible cost-effective care to fight against their prematurity. Flaxseed pillow was effective in reducing the stress of premature infants in the NICU. Such research studies should be encouraged and continued so that the neonatal nurse and caregivers are made aware of the developmental care for premature infants for complete growth and development.

Ethical Clearance- Taken from the Institutional ethics committee. (NUINS/CON/NU/IEC/ 2014-15 dated 26/09/2014).

\section{Conflict of Interest - Nil \\ Source of Funding - Self \\ References}

1. Symington AJ, Pinelli J. Developmental care for promoting development and preventing morbidity in preterm infants. Cochrane database of systematic reviews. 2006(2).

2. Dieter JN, Field T, Hernandez-Reif M, Emory EK, et al. Stable preterm infants gain more weight and sleep less after five days of massage therapy. Journal of pediatric psychology. 2003 Sep 1;28(6):403-11.

3. Als H. A synactive model of neonatal behavioral organization: framework for the assessment of neurobehavioral development in the premature infant and for support of infants and parents in the neonatal intensive care environment. Physical \& Occupational Therapy in Pediatrics. 1986 Jan 1;6(3-4):3-53.

4. AbouseifHA, Mansour AF, Sabbour SF. Prevalence and outcome of Preterm Premature Rupture of Membranes (PPROM) among pregnant women attending Ain Shams maternity hospital. Egyptian Journal of Community Medicine. 2018 Apr;36(2).

5. Kirke AB. How safe is GP obstetrics? An assessment of antenatal risk factors and perinatal outcomes in one rural practice. Rural Remote Health. 2010 Sep 5;10(3):1545.

6. Diesel, Holly Johanna. Soothability and growth in preterm neonates. University of Missouri - Saint Louis, ProQuest, UMI Dissertations Publishing, 2009 March; 3393628. Availablefrom:http://search.proquest.com/ docview/305086190? accountid=148158

7. Diesel HJ, Ercole PM. Soothability and growth in preterm infants. Journal of Holistic Nursing. 2012 Mar;30(1):38-47. 